\title{
MSECO-CERT: Uma Abordagem Baseada em Processo para Apoiar a Certificação de Apps em Ecossistema de Software Móvel
}

\author{
Awdren de Lima Fontão, Arilo Claudio Dias-Neto ${ }^{1}$, Rodrigo Pereira dos Santos ${ }^{2}$ \\ ${ }^{1}$ Programa de Pós-Graduação em Informática (PPGI) \\ Instituto de Computação (ICOMP) - Universidade Federal do Amazonas (UFAM) \\ Caixa Postal 6.200 - 69.077-000 - Manaus - AM - Brasil \\ \{awdren, arilo\}@icomp.ufam.edu.br \\ ${ }^{2}$ DIA - Universidade Federal do Estado do Rio de Janeiro (UNIRIO) \\ Caixa Postal 296 - 22.290-240 - Rio de Janeiro - RJ - Brasil \\ rpseuniriotec.br
}

\begin{abstract}
In a Mobile Software Ecosystem (MSECO), software organizations have restructured their processes to developers aiming to achieve goals, such as increasing the number of mobile applications (apps). However, the Quality Barrier provided by the Apps Store does not set criteria that can ensure that apps certified from them achieve the goals. It is necessary to consider the quality of support offered to the developers through the processes, since working in the processes (certification) can achieve the expected performance for the apps. In this work, a process-based approach to app certification (MSECO-CERT) was defined. The use of MSECO-CERT for app development generated a growth coefficient of downloads 363\% higher and average user ratings $28 \%$ higher compared to an ad hoc approach.
\end{abstract}

Resumo. Em um Ecossistema de Software Móvel (MSECO), as organizações de software passaram a reestruturar seus processos para desenvolvedores visando atingir metas, como o aumento do número de aplicações móveis (apps). No entanto, a "barreira" de qualidade provida pela Loja de Apps, não define critérios que garantem que as apps certificadas a partir deles atinjam as metas. É necessário considerar a qualidade de suporte oferecido aos desenvolvedores por meio dos processos (certificação), para atingir o desempenho esperado para as apps. Neste trabalho, foi definida uma abordagem baseada em processos para certificação de apps (MSECO-CERT). O uso da MSECO-CERT para desenvolvimento de apps gerou um coeficiente de crescimento de downloads $363 \%$ maior e média de avaliações dos usuários $28 \%$ maior em comparação a uma abordagem ad hoc.

\section{Introdução}

Um Ecossistema de Software Móvel (MSECO) consiste de um sistema de evolução cooperativa de aplicações móveis (apps), desenvolvedores e usuários que formam complexos relacionamentos preenchendo nichos, competindo e cooperando, de uma forma similar a ecossistemas biológicos [Lin e Ye, 2009]. Um MSECO é formado por elementos que possuem responsabilidades distintas e que precisam se relacionar de forma harmônica de forma a manter um equilíbrio. Dentre esses elementos, além da 
App, desenvolvedores e usuários, citados anteriormente, podem ser incluídos: a Organização Central, que é responsável pela orquestração do MSECO; os Evangelistas, que são especialistas internos da organização alocados para dar suporte aos desenvolvedores; e, a Loja de Apps, que consiste em um repositório de apps e que possui um papel fundamental para o sucesso do MSECO, definindo políticas de aceitação de apps que irão ser disponibilizadas aos usuários. O desempenho destes elementos precisa ser acompanhado com o objetivo de identificar e prever áreas de melhoria, ou seja, habilidade em suportar e permanecer variável e produtivo durante $o$ tempo. A análise da habilidade se refere ao conceito de "saúde" definido por Manikas e Hansen (2013a).

De forma geral, o impacto de uma app no ecossistema só pode ser medido após a sua publicação em uma loja. Não se pode garantir que apps aprovadas pelos critérios de uma loja resultarão em um número alto de downloads e em avaliações positivas pelos usuários. Desta forma, a submissão de uma app baseada somente em critérios da loja, com foco somente na qualidade do produto, pode causar a "doença" em elementos do MSECO. Isto desencadeia sintomas como a desmotivação dos desenvolvedores para evoluir uma app e a adaptação constante de estratégias pela Organização Central para manter a comunidade de usuários e de desenvolvedores. Então, a prevenção para não gerar impacto negativo na "saúde" do MSECO não deve ser somente focada na qualidade do produto, mas deve levar em consideração a qualidade dos processos executados pelo desenvolvedor (criação e desenvolvimento da app), pela Organização Central (orquestração do ecossistema) e pelo evangelista (suporte e elo entre a organização e o desenvolvedor), uma vez que a "saúde" do MSECO é diretamente influenciada pela saúde dos elementos que o compõe. O problema tratado como foco neste trabalho é o impacto dos elementos para certificar uma app a partir da qualidade dos processos que eles são responsáveis.

Neste trabalho, o conceito de certificação, baseado em Babiy et al. (2010), é interpretado como atestar, por meio da utilização dos processos e do conjunto de práticas/recomendações, que haverá um impacto positivo em indicadores de saúde como a quantidade de downloads e de média de avaliação das apps. A saúde de cada componente do MSECO tem uma relação com práticas de garantia de qualidade e a organização central pode influenciar a garantia de qualidade por meio do estabelecimento de regras e processos a serem seguidos [Axelsson e Skoglund 2016].

Assim, o objetivo deste trabalho é definir e avaliar uma abordagem de certificação de qualidade de apps no contexto de MSECO por meio de processos, recomendações, práticas e indicadores de saúde na fase de pré-publicação para uma loja de apps. Essa abordagem envolve os elementos que compõem o MSECO (desenvolvedor, evangelista, Organização Central, aplicação móvel e loja) a fim de impactar positivamente a saúde do MSECO. Como contribuições para a comunidade científica e para a indústria, apresenta-se os seguintes resultados: Definição da abordagem MSECO-CERT, incluindo os processos de orquestração, desenvolvimento e suporte; Mapeamento Sistemático da Literatura sobre MSECO, incluindo oportunidades de pesquisa; Pacote de estudos experimentais no contexto de ecossistemas de software.

Este artigo é organizado como segue. A próxima seção apresenta a metodologia de pesquisa adotada para a concepção e avaliação da abordagem proposta nesta dissertação de mestrado. A Seção 3 descreve a abordagem MSECO-CERT e seus 
componentes. Na Seção 4 são apresentados os estudos experimentais para a avaliação da abordagem e os resultados obtidos. A Seção 5 apresenta as conclusões incluindo limitações e trabalhos futuros. E, por fim, a seção 6 discorre sobre publicações científicas geradas a partir desta dissertação.

\section{Metodologia de pesquisa}

A metodologia de pesquisa, baseada em Mafra et al. (2006), utilizada neste trabalho é composta por duas fases: concepção, onde o foco é a definição e construção da abordagem MSECO-CERT (Mobile Software Ecosystem App Certification), e; avaliação, focada em avaliar a viabilidade e a utilização da abordagem. Na Figura 1 são apresentadas as etapas dentro de cada fase. Nesta seção, são descritos os estudos que estão inseridos na fase de concepção. $\mathrm{Na}$ Seção 4 , os estudos da fase de avaliação são apresentados.

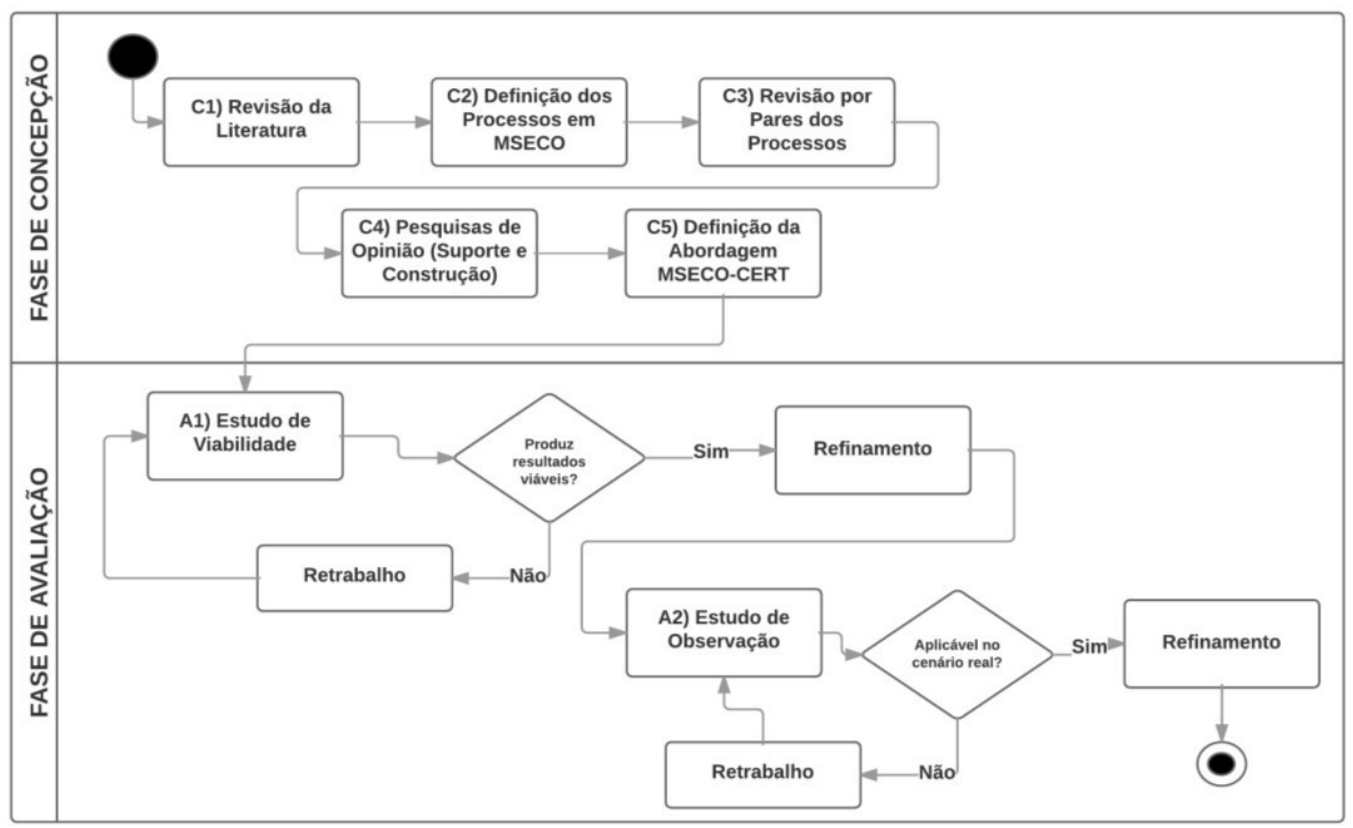

Figura 1. Metodologia de pesquisa para definição da MSECO-CERT

$\mathrm{Na}$ fase de concepção os seguintes estudos foram realizados.

Revisão da Literatura: para esta atividade, foi realizado um mapeamento sistemático com o objetivo de analisar o cenário de MSECO com o propósito de caracterizar em relação aos benefícios, desafios, definições, processos, metodologias, processos e abordagens do ponto de vista do pesquisador no contexto de pesquisas acadêmicas em engenharia de software.

Este mapeamento sistemático, a partir da análise dos estudos selecionados, apontou a necessidade por abordagens para apoiar atividades em MSECO. Os estudos selecionados também foram analisados para extrair recomendações e práticas que pudessem ser aplicados às atividades do processo definido nesta dissertação.

Definição dos Processos em MSECO: definição inicial dos processos que estruturam as atividades dentro de um MSECO com o objetivo de entender as atividades, elementos e artefatos envolvidos quando o cenário de desenvolvimento da aplicação é um ecossistema. As atividades são compostas de: 
- Definição de recomendações para cada atividade, como forma de suportar a execução da atividade. Essas recomendações fazem parte da composição de uma atividade em cada processo;

- Definição de práticas para cada atividade, com o objetivo de impactar positivamente na saúde do MSECO. Essas práticas estão associadas à cada atividade dentro dos processos

Esta etapa permitiu a definição inicial de três processos: MSECO-ORQ (processo de orquestração), MSECO-SUP (processo de suporte) e MSECO-DEV (processo de desenvolvimento). A notação utilizada para a modelagem dos processos foi baseada na notação proposta por Villela et al. (2004).

Revisão por Pares dos Processos: realizada com especialistas (Organização Central, evangelistas e desenvolvedores) para avaliar se os processos (orquestração, suporte e construção) especificados neste trabalho estão adequados para atender às necessidades de orquestração, desenvolvimento de aplicações móveis e evangelismo, do ponto de vista de profissionais com experiência nas áreas de conhecimento dos processos.

A revisão por pares auxiliou no levantamento de sugestões para a evolução dos processos que compõem a MSECO-CERT. A maioria das sugestões (que foram totalmente ou parcialmente aceitas) se concentrou em: descrição de atividades (11 sugestões $-28,95 \%$ ), artefatos consumidos e produzidos por atividades (11 sugestões $28,95 \%$ ) e papéis participantes em atividades ( 8 sugestões $-21,05 \%$ ). Dentro dos perfis dos revisores foi possível perceber ainda a participação deles em mais de um MSECO, logo a revisão pode partir da experiência de contribuições para MSECO da Google, Microsoft, Apple, Nokia e Samsung.

Pesquisas de Opinião: baseando-se no relatório técnico publicado em Travassos et al. (2002), pesquisas de opinião são estudos para analisar as atividades, práticas e recomendações que compõem os processos de construção e de suporte. Nesta atividade, foram realizadas as seguintes pesquisas de opinião:

- Com evangelistas para analisar práticas e recomendações que compõem o processo de suporte com o propósito de caracterizar com respeito à aplicabilidade do ponto de vista dos evangelistas no contexto de atividades de engajamento e treinamento em MSECO;

Nenhuma prática foi removida, 7 novas práticas foram adicionadas e práticas e recomendações associadas às atividades de evangelismo em MSECO foram ajustadas, consolidando um processo de suporte com 43 práticas e 9 recomendações.

- Com desenvolvedores experientes para analisar as recomendações e práticas que compõem o processo de construção com o propósito de caracterizar com respeito à utilidade do ponto de vista de desenvolvedores experientes no contexto de atividades de desenvolvimento em MSECO;

- Com desenvolvedores novatos para analisar um subconjunto de práticas e atividades que compõem o processo de construção com o propósito de caracterizar com respeito a utilidade e aplicabilidade do ponto de vista de desenvolvedores novatos no contexto de atividades de desenvolvimento de aplicações móveis em MSECO. 
Do ponto de vista dos desenvolvedores experientes 4 práticas precisaram de ajustes que foram realizados. Além disso, eles indicaram 3 novas práticas que envolvem uso de bibliotecas de terceiros, experiência do usuário e análise de concorrentes. Os desenvolvedores novatos indicaram a necessidade de ajuste na sequência das atividades e que algumas práticas poderiam ser antecipadas.

Nesta seção foram descritos os estudos que ajudaram na concepção da MSECOCERT. Conforme descrito acima, quatro estudos foram realizados na fase de concepção: mapeamento sistemático da literatura, revisão por pares, pesquisa de opinião com evangelistas, pesquisa de opinião com desenvolvedores (experientes e novatos). Esses estudos permitiram ajustes nas atividades (incluindo descrição, artefatos e critérios de entrada e saída), nas práticas e nas recomendações. Além disso, esses estudos permitiram adequar a abordagem ao cotidiano de evangelistas e desenvolvedores de aplicações móveis. A próxima seção apresenta a MSECO-CERT na versão após as fases de concepção e avaliação.

\section{Abordagem MSECO-CERT}

A abordagem MSECO-CERT (Figura 1) é composta por três processos MSECO-ORQ (orquestração), MSECO-SUP (suporte) e MSECO-DEV (desenvolvimento), a descrição completa dos processos está disponível no Capítulo 3 da dissertação.
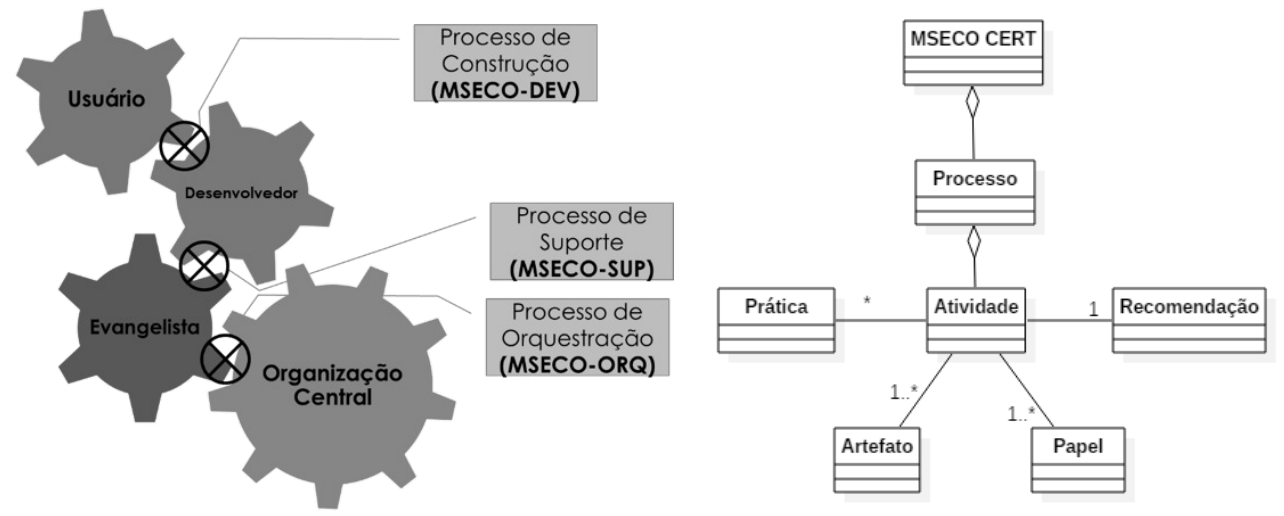

Figura 2. Relação entre processos (Esq.) e estrutura da MSECO-CERT (Dir.)

Como mostrado na Figura 1, cada um dos processos é composto por atividades, papéis o que envolve um fluxo de trabalho utiliza e produz artefatos. Para cada atividade, foi associada uma recomendação, orientação para executá-la. Para cada recomendação, práticas, que representam exercícios para atingir resultados concretos nas metas organizacionais, foram associadas. As práticas só estão associadas às atividades realizadas antes da submissão de uma app à loja, pois é quando o evangelista pode intervir. A MSECO-CERT é composta como segue.

MSECO-ORQ: surge da interação entre a organização central e o evangelista, o objetivo deste processo é preparar, gerenciar e coordenar alguns elementos (desenvolvedor, evangelista e a app) [Jansen et al., 2009] e alguns de seus relacionamentos. Além disso, visa fornecer diretrizes e guias necessários com o objetivo de manter os indicadores de saúde de MSECO: robustez, produtividade e criação de nicho. Possui nove atividades, nove artefatos, nove recomendações, nenhuma prática (uma vez que não tem como gerar intervenção na orquestração a partir dos papéis de evangelista e desenvolvedor). 
Na Figura 2 e Tabela 1 é apresentado um exemplo de atividade que compõe o MSECO-ORQ, especificamente a atividade 2: Disponibilizar os Guias de Interface. Os documentos gerados ao final deste processo formam a base do MSECO, que compõem, ao final do processo, o artefato Diretrizes do MSECO, que servirá para outros processos que compõem esta abordagem. Esses documentos são: Especificação da Plataforma, Guias de Design e Interface, Guias e Ferramentas de Marketing, Ferramentas de Desenvolvimento, Central do Desenvolvedor, Loja de Aplicações, Critérios da Loja.

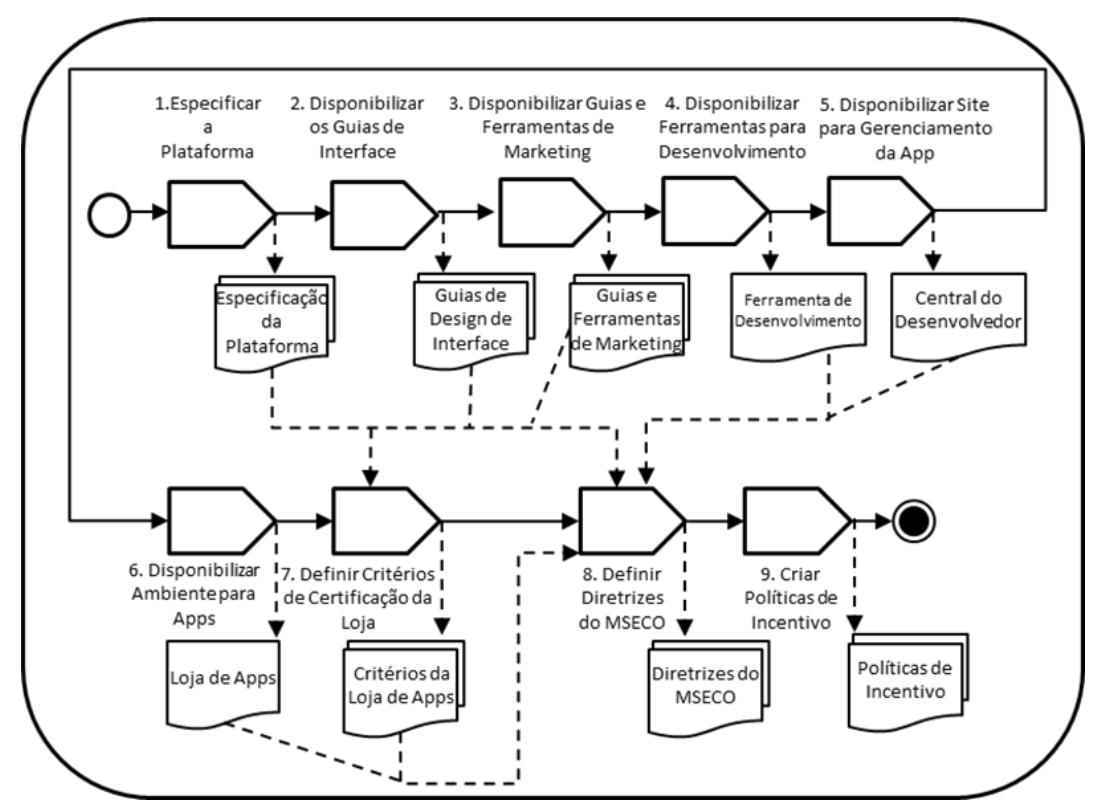

Figura 2. MSECO-ORQ: Processo de Orquestração

Tabela 1. MSECO-ORQ: Atividade 2

\begin{tabular}{|l|l|}
\hline Atividade: & 2 Disponibilizar Guias de Design e Interface de Usuário da Plataforma \\
\hline Descrição: & $\begin{array}{l}\text { A interface de usuário de uma plataforma é sua identidade. É importante divulgar guias } \\
\text { que ajudem os desenvolvedores a criar e desenvolver soluções que respeitem os padrões de } \\
\text { design e interface da plataforma do MSECO. Essa atividade tem como objetivo } \\
\text { disponibilizar a base necessária para manter a boa interação com o usuário e o padrão da } \\
\text { plataforma do MSECO. }\end{array}$ \\
\hline $\begin{array}{l}\text { Critérios de } \\
\text { Entrada: }\end{array}$ & Ter-se a Especificação da Plataforma pronta ou atualizada. \\
\hline Critérios de Saída: & Documentos com especificação de interface e interação da plataforma criados e aprovados. \\
\hline Responsável: & Organização Central \\
\hline Participantes: & Equipe de Design e Equipe de UX (Experiência do Usuário) da Organização. \\
\hline $\begin{array}{l}\text { Artefatos } \\
\text { Requeridos: }\end{array}$ & Especificação da Plataforma. \\
\hline $\begin{array}{l}\text { Artefatos } \\
\text { Produzidos: }\end{array}$ & Guias de Design e Interface de Usuário, e Aplicações Móveis de Referência. \\
\hline Recomendação: & $\begin{array}{l}\text { Deve-se levar em consideração as limitações e potencialidades da plataforma na execução } \\
\text { desta atividade. }\end{array}$ \\
\hline
\end{tabular}

MSECO-SUP: o objetivo deste processo é prover o relacionamento entre a organização central com os desenvolvedores. Para isso, o MSECO dispõe de um elemento responsável por esta ligação: o evangelista. Contém nove atividades e 
recomendações, seis artefatos, quarenta e quatro práticas. Na Figura 3 e Tabela 2 é apresentado um exemplo de atividade deste processo.

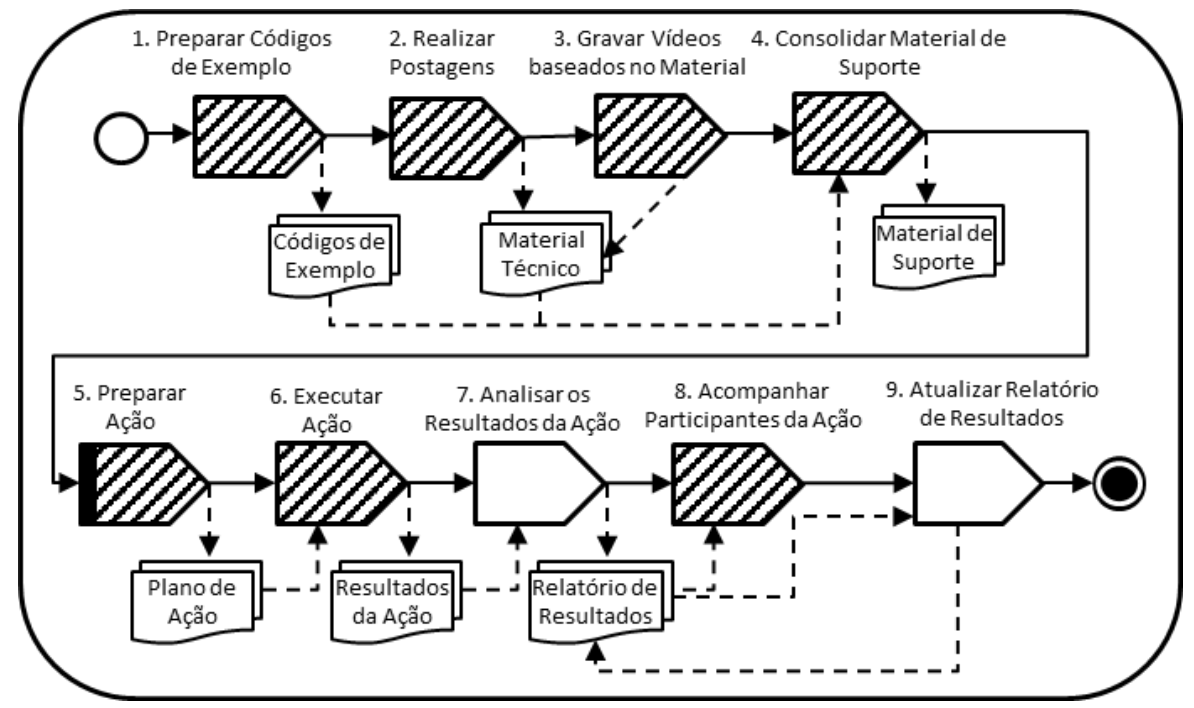

Figura 3. MSECO-SUP: Processo de Suporte

As atividades visam estabelecer um procedimento para a execução do trabalho dos evangelistas dentro dos padrões da organização, por meio do artefato Diretrizes do $M S E C O$. Os documentos gerados ao final deste processo formam a base de divulgação do MSECO para os desenvolvedores externos e estabelecem procedimentos de relacionamento com estes desenvolvedores.

Esta atividade visa ajudar na divulgação dos artefatos gerados ainda no processo de orquestração, fazendo com que esses artefatos sejam facilmente acessados e entendidos pelos desenvolvedores internos e os externos. A marcação diferenciada em algumas atividades indica que pode-se intervir nas atividades com o objetivo de gerar algum impacto na saúde do MSECO. Abaixo apresentamos um exemplo de atividade que compõe o MSECO-SUP, especificamente a atividade 3: Gravar Vídeos baseados no Material. A descrição de todo o processo está disponível no Capítulo 3, Seção 3.3.2 da dissertação.

Tabela 2. MSECO-SUP: Atividade 3

\begin{tabular}{|l|l|}
\hline Atividade: & 3 Gravar Vídeos baseados no Material \\
\hline Descrição: & $\begin{array}{l}\text { Esta atividade tem como objetivo explicar conceitos, dicas, implementação e utilização de } \\
\text { ferramentas de uma forma mais interativa. }\end{array}$ \\
\hline Critérios de Entrada: & $\begin{array}{l}\text { Deve-se definir um roteiro para a gravação do vídeo, pode ser ainda vídeos gerados em } \\
\text { cima de material em Wiki ou blogs. }\end{array}$ \\
\hline Critérios de Saída: & O vídeo deve conter informações práticas e que possam ser replicadas. \\
\hline Responsável: & Evangelista. \\
\hline Participantes: & $\begin{array}{l}\text { Equipe de Marketing de Desenvolvedores. Algum outro desenvolvedor (interno ou } \\
\text { externo) pode participar. }\end{array}$ \\
\hline Artefatos Requeridos: & Diretrizes do MSECO, Material Técnico. \\
\hline Artefatos Produzidos: & Material Técnico. \\
\hline Recomendação: & $\begin{array}{l}\text { Os vídeos devem fornecer experiência prática de algum conteúdo do MSECO. Algo que } \\
\text { possa ser seguido ou uma dica. É importante que no vídeo sejam ainda indicados links } \\
\text { para postagens e documentos referentes ou similares ao conteúdo do vídeo. }\end{array}$ \\
\hline $\begin{array}{l}\text { Como impactar na } \\
\text { certificação de } \\
\text { aplicações: }\end{array}$ & $\begin{array}{l}\text { P19-S } \\
\text { produzir vídeos detalhados sobre código desenvolvido na Atividade 1 e sobre as } \\
\text { postagens feitas na Atividade 2 para guiar o desenvolvedor durante processo de } \\
\text { desenvolvimento. }\end{array}$ \\
\hline
\end{tabular}


MSECO-DEV: o objetivo deste processo é que o desenvolvedor planeje e construa uma app que será adicionada à loja de apps. Para isso, o desenvolvedor poderá utilizar artefatos gerados tanto no MSECO-ORQ como no MSECO-SUP. Dessa forma, ele pode contribuir com a produtividade e a criação de nicho do MSECO. Contém oito atividades e recomendações, sete artefatos, dezesseis práticas.

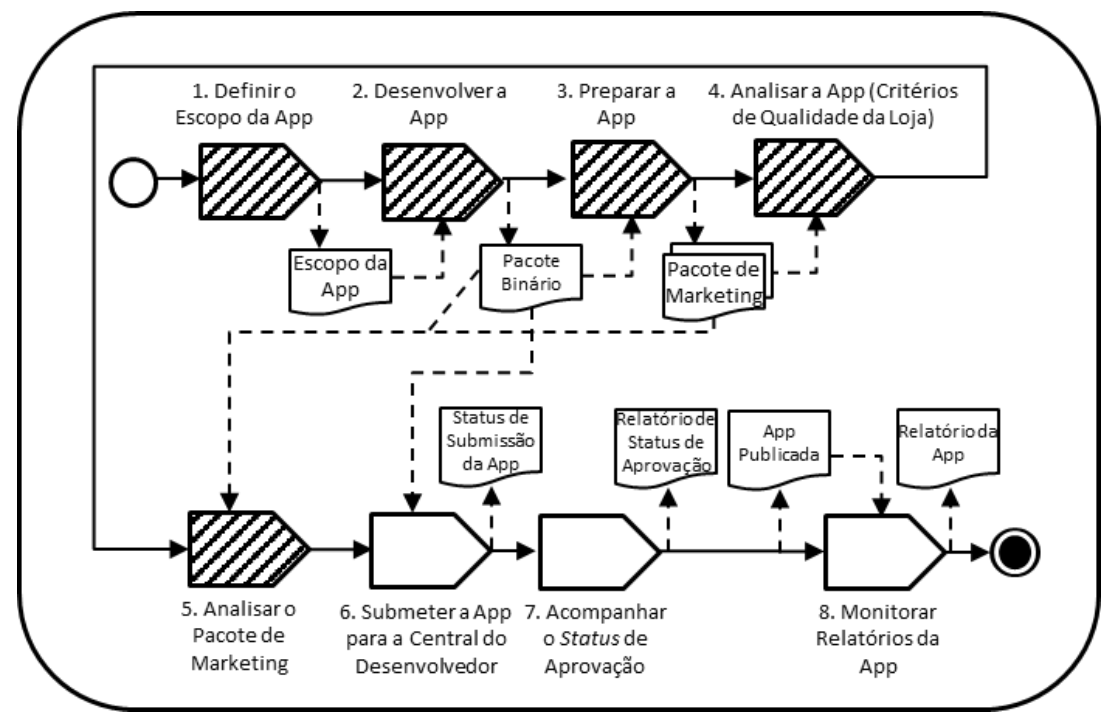

Figura 3. MSECO-DEV: Processo de Desenvolvimento

Na Figura 3 e Tabela 3 é apresentado um exemplo de atividade que compõe o MSECO-DEV, especificamente a atividade 5: Analisar o Pacote de Marketing. A descrição de todo o processo está disponível no Capítulo 3, Seção 3.3.2 da dissertação.

Tabela 3. MSECO-DEV: Atividade 5

\begin{tabular}{|c|c|}
\hline Atividade: & 5 Analisar o Pacote de Marketing \\
\hline Descrição: & $\begin{array}{l}\text { Analisar o pacote de marketing preparado juntamente com o binário final da aplicação e } \\
\text { guias de marketing do MSECO. Visa, assim, validar se houve alguma mudança na } \\
\text { aplicação durante a execução das Atividades } 1.3 \text { e 1.4. }\end{array}$ \\
\hline $\begin{array}{l}\text { Critérios de } \\
\text { Entrada: }\end{array}$ & A aplicação deve estar pronta e analisada sobre os critérios da loja. \\
\hline Critérios de Saída: & Pacote de Marketing da Aplicação consolidado. \\
\hline Responsável: & Desenvolvedor. \\
\hline Participantes: & Evangelista. \\
\hline $\begin{array}{l}\text { Artefatos } \\
\text { Requeridos: }\end{array}$ & Material de Marketing da Aplicação, Material de Suporte e Binário da Aplicação móvel. \\
\hline $\begin{array}{l}\text { Artefatos } \\
\text { Produzidos: }\end{array}$ & Pacote de Marketing da Aplicação. \\
\hline Recomendação: & $\begin{array}{l}\text { Nesta atividade, as imagens das telas da aplicação podem ser adicionadas ao pacote de } \\
\text { marketing. Planejar estratégias de marketing. }\end{array}$ \\
\hline $\begin{array}{l}\text { Como impactar a } \\
\text { certificação de } \\
\text { aplicaçôes: }\end{array}$ & \begin{tabular}{l|l} 
P16-C & $\begin{array}{l}\text { Utilizar um checklist para assegurar a completude do material de marketing } \\
\text { gerado e sua adequação com os requisitos de marketing da loja. }\end{array}$
\end{tabular} \\
\hline
\end{tabular}

Neste processo, as atividades estão relacionadas a prover informações e permitir o desenvolvimento de uma aplicação móvel. Durante a execução das atividades, o desenvolvedor pode contar ainda com a ajuda do evangelista.

Um exemplo de utilização da MSECO-CERT envolve o treinamento conduzido por um evangelista que faz uso do MSECO-SUP para preparar códigos de exemplo, realizar postagens, gravar vídeos, consolidar material de suporte, preparar ação (evento ou treinamento), executar ação, analisar os resultados da ação, acompanhar os 
participantes e atualizar relatórios de resultados. Os documentos gerados ao final formam a base de divulgação do MSECO e fornecem procedimentos de relacionamento com os desenvolvedores.

Os desenvolvedores utilizam o MSECO-DEV para definir o escopo da app, desenvolve-la, preparar material de divulgação, analisar a app (critérios de qualidade da loja), analisar pacote de marketing, submeter a app, acompanhar status de aprovação e monitorar relatórios. $\mathrm{O}$ artefato gerado ao final desse processo deve ser um arquivo publicável empacotado, usando o padrão definido pelo MSECO e que deve ser suportado pela sua plataforma. Este artefato poderá ser embarcado no dispositivo de um usuário, pois estará disponível na loja de apps.

\section{Experimentos e Resultados}

A abordagem, baseada em Shull et al. (2001), foi avaliada a partir de dois aspectos : 1) Eficiência da abordagem (Estudo de viabilidade) - o propósito foi responder à seguinte questão: "A utilização da MSECO-CERT para certificar apps desenvolvidas no contexto de MSECO é viável analisando a sua eficiência em relação à quantidade de downloads e à avaliação das apps desenvolvidas?"; 2) Adequação em um cenário real (Estudo de observação) - a seguinte questão foi investigada: " $A M S E C O-C E R T$ é adequada no contexto real para o evangelismo e desenvolvimento de apps?".

Tendo em vista que uma modificação na orquestração de um MSECO não é um processo que pode ser executado rapidamente e está associado à estratégia da organização central, não foi avaliado o uso do processo MSECO-ORQ nos experimentos, pois o fluxo de atividades do processo permanece o mesmo em relação aos três principais ecossistemas e não se modifica com frequência. Nos experimentos, focou-se na avaliação dos processos MSECO-DEV e MSECO-SUP.

\subsection{Estudo de viabilidade - Eficiência da abordagem}

O objetivo é esquematizado a partir do paradigma GQM [Basili et al., 1994], como segue: Analisar a aplicação da abordagem MSECO-CERT com o propósito de caracterizá-la em relação à eficiência do ponto de vista dos pesquisadores no contexto de engajamento e treinamento de desenvolvedores de aplicações móveis e evangelistas no cumprimento de metas organizacionais em MSECO. Como forma de atingir esse objetivo foram definidas as seguintes questões e métricas:

Q1. Qual é a eficiência da abordagem MSECO-CERT em relação a uma abordagem $A d$ hoc no que diz respeito à quantidade de downloads de aplicações móveis?

Métrica: Quantidade de downloads de aplicações móveis desenvolvidas pelos participantes;

Q2. Qual é a eficiência da abordagem MSECO-CERT em relação a uma abordagem $A d$ hoc no que diz respeito à avaliação de aplicações móveis geradas?

Métrica: Média de avaliação de aplicações móveis desenvolvidas pelos participantes.

Com os resultados obtidos por meio da execução do estudo de viabilidade foi possível caracterizar a MSECO-CERT em relação a uma abordagem Ad hoc que foi aplicada por não ter sido identificado na literatura outra abordagem similar à MSECOCERT. Este estudo envolveu dois evangelistas oficiais do MSECO gerenciado pela 
Microsoft para a plataforma Windows Phone. Trinta desenvolvedores (divididos em dois grupos com quinze participantes cada) foram provenientes dos cursos de Engenharia de Software e Sistemas de Informação do Instituto de Ciências Exatas e Tecnologia da UFAM. Apesar de o cenário envolver uma universidade, este é o cenário real, pois organizações que treinam desenvolvedores buscam universidades para oferecer treinamentos oficiais.

O estudo consistiu na execução do treinamento e acompanhamento dos desenvolvedores. Para a execução do treinamento a plataforma utilizada foi a Windows Phone 7.5, com o ambiente Visual Studio 2012. Desta forma, com a Turma 1 foi aplicada a abordagem MSECO-CERT, o evangelista utilizou o MSECO-SUP e os desenvolvedores utilizaram o MSECO-DEV. Enquanto que com a Turma 2 a abordagem $A d$ hoc foi utilizada, tanto para o evangelismo quanto o desenvolvimento. $\mathrm{O}$ estudo em um ambiente acadêmico, está dentro do contexto real de uma atividade de evangelismo e desenvolvimento em um MSECO, este cenário é o utilizado pelas organizações em MSECO, o que caracteriza um ambiente real, com a mesma infraestrutura, mesmas ferramentas e um evangelista para cada turma.

A mesma ementa de treinamento foi utilizada para as duas turmas, considerando um curso básico: a) conceitos de desenvolvimento de aplicações móveis; b) ferramentas de desenvolvimento; c) padrões de desenvolvimento; d) interface de usuário; e) classes, métodos e comportamento da aplicação móvel; f) depuração de código; g) empacotamento de aplicação móvel; h) publicação de aplicações móveis. E as duas turmas tinham como meta o desenvolvimento e a publicação da aplicação móvel. A carga horária de treinamento para as duas turmas foi de 18 horas. Após a execução do treinamento, onde os desenvolvedores iniciaram a construção das suas aplicações móveis, os desenvolvedores responderam ao Pós-Questionário para entender a mudança em atitudes gerais e a comparação entre atitudes específicas de cada turma. Após isto, os evangelistas iniciaram uma fase de acompanhamento dos desenvolvedores, durante duas semanas após o treinamento. Para isto, dois grupos no WhatsApp e Facebook foram criados para a Turma 1 e Turma 2. Os evangelistas ainda realizaram suporte por e-mail. O acompanhamento foi realizado com o objetivo de concluir o desenvolvimento de aplicações móveis e submete-las a loja de aplicações móveis

Os dados de quantidade de downloads foram coletados diretamente da Central do Desenvolvedor ${ }^{1}$ durante um período de 30 a partir da publicação de cada aplicação móvel. Este procedimento foi realizado para as duas turmas. Como forma de realizar uma comparação entre as duas turmas, calculou-se a média diária de downloads das aplicações móveis. Como esses valores foi gerada por dia a média acumulada, estes dados são apresentados na Tabela 4. A partir dos resultados obtidos dos downloads, foi realizada uma comparação entre as funções que descrevem o crescimento do número de downloads do conjunto de aplicações de cada turma, onde $f(x)=8,809 x-3,8138$ (Turma 1) e $g(x)=1,9591 x-1,2322$ (Turma 2). Uma vez que uma função afim pode ser descrita como $h(x)=a x+b$, onde $a$ é o coeficiente de crescimento da reta, podemos observar que o coeficiente de crescimento da função da turma $1(8,80)$ é $363 \%$ maior que o da turma $2(1,95)$. Isto aponta uma diferença entre o indicador de downloads, no qual a turma 1 obteve uma média acumulada maior.

\footnotetext{
${ }^{1}$ https://developer.microsoft.com/pt-br/dashboard/apps/overview
} 
Tabela 4. Média acumulada por dia de downloads

\begin{tabular}{|c|c|c|c|c|c|c|c|c|}
\hline \multirow{2}{*}{ Dia } & \multicolumn{2}{|c|}{ Turma } & \multirow{2}{*}{ Dia } & \multicolumn{2}{|c|}{ Turma } & \multirow{2}{*}{ Dia } & \multicolumn{2}{|c|}{ Turma } \\
\hline & 1 & 2 & & 1 & 2 & & 1 & 2 \\
\hline $\mathbf{1}^{\circ}$ & 4 & 2 & $11^{\circ}$ & 93 & 20 & $21^{\circ}$ & 181 & 40 \\
\hline $2^{\circ}$ & 11 & 5 & $12^{\circ}$ & 103 & 21 & $22^{\circ}$ & 188 & 42 \\
\hline $3^{\circ}$ & 18 & 6 & $13^{\circ}$ & 113 & 22 & $\mathbf{2 3}^{\circ}$ & 196 & 43 \\
\hline $4^{\circ}$ & 28 & 8 & $14^{\circ}$ & 122 & 24 & $24^{\circ}$ & 207 & 45 \\
\hline $5^{\circ}$ & 38 & 9 & $15^{\circ}$ & 132 & 25 & $25^{\circ}$ & 214 & 49 \\
\hline $6^{\circ}$ & 49 & 10 & $16^{\circ}$ & 141 & 27 & $26^{\circ}$ & 221 & 51 \\
\hline $7^{\circ}$ & 61 & 13 & $17^{\circ}$ & 149 & 33 & $27^{\circ}$ & 230 & 53 \\
\hline $8^{\circ}$ & 68 & 14 & $18^{\circ}$ & 158 & 34 & $28^{\circ}$ & 241 & 55 \\
\hline $9^{\circ}$ & 76 & 16 & $19^{\circ}$ & 166 & 37 & $29^{\circ}$ & 252 & 56 \\
\hline $10^{\circ}$ & 84 & 18 & $20^{\circ}$ & 173 & 38 & $30^{\circ}$ & 267 & 58 \\
\hline
\end{tabular}

Como forma de testar a hipótese nula A (HO A: A quantidade de downloads das aplicações móveis desenvolvidas utilizando a abordagem MSECO-CERT em comparação com quantidade de downloads das aplicações desenvolvidas com a abordagem Ad hoc são similares), seguiram-se os procedimentos para testes estatísticos. Os dados seguem distribuição normal a partir do teste de normalidade Shapiro-Wilk. Considerando um nível de confiança de $95 \%(a=0,05)$, utilizou-se o teste $t$-student (pareado e bicaudal) para testar a hipótese. O p-value é menor que 0.00001, logo resultado é significante para $p<0.05$ e a hipótese H0 A é rejeitada (a comparação da quantidade de downloads entre as duas turmas não é similar). Para analisar a média das avaliações de cada turma, foram levadas em consideração as avaliações das aplicações móveis que obtiveram alguma classificação pelos usuários. Com esta informação, calculou-se a média, mediana de avaliação para cada turma e o desvio padrão. O desviopadrão da avaliação de aplicações da Turma $1(0,5)$ é menor que a da turma $2(1,7)$. Quando se volta a atenção para a tendência das avaliações, as avaliações da turma 1 são mais próximas de 4,5. Já as avaliações da Turma 2 se dividem em uma aproximação entre 4,3 e 2,5 .

Em relação a análise qualitativa, na seção 5.5.2 da dissertação são apresentados detalhes da análise da planilha de acompanhamento do processo de desenvolvimento utilizado pelos desenvolvedores da turma 1, das anotações durante a utilização do processo MSECO-SUP pelo evangelista da turma 1, incluindo observações acerca do MSECO-DEV a partir da observação deste evangelista. Ainda foram analisas qualitativamente as respostas de uma entrevista semiestruturada com o evangelista da turma 2 após o treinamento e o acompanhamento dos desenvolvedores, as respostas do formulário pós-treinamento tanto da turma 1 quanto da turma 2, e conversas por e-mail, WhatsApp e Facebook, a partir da visão de DX (Experiência de Desenvolvedor).

Mesmo com os resultados viáveis e que confirmam a aplicabilidade da abordagem, percebeu-se a necessidade de refinar a forma de apresentar a abordagem e, também, de refinar a sequência de práticas e descrição de atividades, assim como, disponibilizar o conjunto de artefatos necessários para a utilização da abordagem.

\subsection{Estudo de observação - Adequação em um cenário real}

O objetivo deste estudo estruturado com o GQM foi de: Analisar a aplicação da abordagem MSECO-CERT com o propósito de avaliar em relação à adequação em um contexto real do ponto de vista dos desenvolvedores e do evangelista no contexto de 
engajamento e treinamento de desenvolvedores de aplicações móveis e evangelistas no cumprimento de metas organizacionais em MSECO.

No estudo de observação, como forma de inserir a utilização da abordagem em um cenário real da indústria, o objetivo não foi comparar com a utilização de uma outra abordagem, mas sim obter informações sobre as dificuldades dos participantes e realizar uma análise qualitativa da utilização da MSECO-CERT. Neste estudo, ainda no MSECO Windows Phone, a plataforma utilizada foi a Windows Universal Platform $(U W P)$, optou-se pela mudança para analisar a adequação da abordagem em um cenário onde é comum a atualização das plataformas. Destaca-se o fato de que o evangelista participante é líder da comunidade de desenvolvedores Windows e Android e possui nível alto de experiência (inclusive na indústria) em projeto, desenvolvimento e publicação de apps, assim como, em evangelismo de comunidades de desenvolvedores.

O evangelista foi questionado sobre o grau de dificuldade da utilização do MSECO-SUP para o evangelismo no contexto de MSECO. Este evangelista classificou a utilização como fácil e em relação aos aspectos que tornam o MSECO-SUP de fácil aplicação comentou: "Fácil de usar por ser de fácil entendimento, por tornar o processo de desenvolvimento de apps mais organizado e estabelecer uma metodologia durante todo o trabalho. De primeira a gente sente um pouco de dificuldade em seguir, mas é questão de costume, sempre fazemos de qualquer jeito. ". Quando questionado acerca de que maneira o MSECO-SUP o auxiliou no evangelismo comentou: "Positivamente. O MSECO-SUP me auxiliou no evangelismo de desenvolvedores que executaram atividades de desenvolvimento, publicação e acompanhamento de uma app. Talvez não tivesse chegado ao mesmo resultado se não tivesse o utilizado. ".

Os desenvolvedores também classificaram o grau de dificuldade da aplicação do processo MSECO-DEV, sete (70\%) deles consideram fácil enquanto que $3(30 \%)$ acharam difícil. Entretanto, quando questionados sobre de que maneira o MSECO-DEV o auxiliou no desenvolvimento, publicação e acompanhamento da sua app, todos os dez desenvolvedores $(100 \%)$ responderam que foi de forma positiva, ou seja, o MSECODEV auxiliou no desenvolvimento, publicação e acompanhamento da aplicação móvel e que talvez não tivessem chegado ao mesmo resultado se não tivesse o utilizado. E todos os desenvolvedores complementaram informando que utilizariam o MSECO-DEV para desenvolver suas aplicações móveis futuras.

\section{Considerações finais}

A organização central deve fazer com que o MSECO permaneça saudável, ou seja, produtivo, favorecendo a criação de nichos de usuários e desenvolvedores e que suporte variações de mercado. Para ter controle sobre a análise da saúde, a organização central dispõe de indicadores de saúde como: quantidade de downloads e avaliação média de aplicações móveis por usuários na loja de aplicações móveis. Desta forma o evangelista deve ajudar o desenvolvedor a contribuir para um impacto positivo nesses indicadores, o que impactará positivamente a saúde do MSECO.

Tendo em vista o cenário apresentado, este trabalho de pesquisa definiu e avaliou uma abordagem baseada em processo para apoiar a certificação de aplicações móveis em MSECO. Neste trabalho certificação está associada a utilização dos processos para o evangelismo de desenvolvedores e desenvolvimento de aplicações móveis. Três processos com um conjunto de recomendações associadas às atividades 
foram definidos: MSECO-ORQ (orquestração, seguido pela organização central), MSECO-SUP (suporte, seguido pelo evangelista) e MSECO-DEV (construção, seguido pelo desenvolvedor). Todos passaram por revisão por pares com especialistas, onde foi possível perceber que somente no MSECO-SUP e no MSECO-DEV seria possível intervir para gerar algum impacto nos indicadores de saúde.

Para o MSECO-SUP e o MSECO-DEV foi definido ainda um conjunto de práticas como forma de gerar um resultado concreto (impacto positivo) nos indicadores de saúde do MSECO. Esses dois processos foram analisados por meio de pesquisas de opinião, o MSECO-SUP com evangelistas dos três principais MSECOs (Android, iOS e Windows Phone) e o MSECO-DEV por desenvolvedores novatos e experientes. Estas pesquisas de opinião apoiaram no ajuste de atividades, recomendações e práticas.

Com isso a abordagem MSECO-CERT saiu da etapa de concepção para ser, inicialmente, caracterizada em relação a viabilidade dos resultados produzidos. Um estudo de viabilidade foi executado e percebeu-se que a utilização da abordagem MSECO-CERT em comparação a uma abordagem $A d$ hoc, produz impactos notáveis na quantidade de downloads e na média de avaliações das aplicações móveis. Foi possível ainda perceber a mudança de atitudes positivas dos desenvolvedores que utilizaram a abordagem MSECO-CERT. Este estudo ajudou, também, no refinamento da MSECOCERT.

Após o estudo de viabilidade, um estudo de observação foi executado para identificar as possíveis dificuldades da utilização da abordagem no cenário real. Neste estudo os participantes, evangelista e desenvolvedores, confirmaram que a utilização da abordagem os ajudou positivamente e que talvez não chegassem nos mesmos resultados caso não tivessem a utilizado. Os participantes ainda afirmaram que utilizariam a abordagem para suas próximas atividades (evangelismo e desenvolvimento de aplicações móveis).

Além da experiência relatada para definição e avaliação dos processos, que pode servir de base para outras pesquisas em Ecossistemas de Software, as principais contribuições desta pesquisa são: (1) Definição da abordagem MSECO-CERT; (2) A análise de métricas, extraídas de um conjunto indicado pela literatura existente, para analisar indicadores de saúde de MSECO; (3) Mapeamento Sistemático da Literatura sobre MSECO, incluindo oportunidades de pesquisa; (4) Pacote de estudos experimentais no contexto de ecossistemas de software. A parceria com a indústria desse trabalho se mostra presente com o treinamento de plataformas da Microsoft, em ambientes reais de treinamento, e o envolvimento de evangelistas da Apple, Google e Microsoft em fases de concepção e/ou avaliação da MSECO-CERT.

As limitações desta pesquisa estão relacionadas ao tipo de MSECO, comunidade de desenvolvedores e perfis dos evangelistas, como segue: Os estudos foram realizados apenas no MSECO Windows Phone, então há a necessidade de realizar estudos nos MSECOs Android (em andamento como colaboração) e iOS; Os evangelistas participantes são oficiais do MSECO Windows Phone e um é evangelista oficial do Android, no entanto é necessário ainda avaliar a utilização da abordagem com diferentes níveis de perfil técnico quanto de temperamento psicológico. Apesar de o perfil técnico básico de um evangelista nos três principais MSECOs ser similar. 


\section{Publicações e Colaboração}

Este trabalho gerou cinco publicações de autoria própria e sete publicações em colaboração com outras instituições em conferências e, também, participações em orientação de quatro Trabalhos de Conclusão de Curso. Alguns dos artigos: Mobile Software Ecosystems (MSECO): a systematic mapping study (COMPSAC 2015 Qualis A2); MSECO-SUP: Support Process in Mobile Software Ecosystems (SBES 2015 - Qualis B2); MSECO-DEV: Application Development Process in Mobile Software Ecosystems (SEKE 2016 - Qualis B1); MSECO Skill: Construção de Competências de Desenvolvedores em Ecossistemas de Software Móvel (CIbSE 2014 Qualis B4); Research Opportunities for Mobile Software Ecosystems (WDES 2015 Qualis B5).

Além disso três artigos para periódicos ou conferências em processo de finalização para submissão: An Empirical Evaluation of a Process-Based Approach to Certify Apps in Mobile Software Ecosystems (será submetido ao Journal Information and Software Technology - Qualis A2); A Family of Experiments to Concept and Evaluate a Mobile Application Certification Approach (será submetido ao Empirical Software Engineering - Qualis B1); Mobile Application Development Training in Mobile Software Ecosystem (MSECO): Investigating the Developer Experience (submetido ao SBIE - Qualis B1).

\section{Referências}

Axelsson, J. and Skoglund, M. (2016). Quality assurance in software ecosystems: A systematic literature mapping and research agenda. In: Journal of Systems and Software, v. 114, n. 1, p. 69-81.

Babiy, V., Janicki, R., Wassyng, A., Bogobowicz, A. D. and Wyszynski, S. (2010). Selecting the best strategy in a software certification process. In: Proceedings of the International Multiconference on Computer Science and Information Technology, pp. 53-58.

Jansen, S., Brinkkemper, S. and Finkelstein, A. (2009). Business Network Management as a Survival Strategy: A Tale of Two Software Ecosystems. n. 2, pp. 34-48.

Lin, F. L. F. and Ye, W. Y. W. (2009). Operating System Battle in the Ecosystem of Smartphone Industry. In: Proceedings of the International Symposium on Information Engineering and Electronic Commerce, pp. 617-621.

Mafra, S., Barcelos, R., Travassos, G. (2006). Aplicando uma metodologia baseada em evidência na definição de novas tecnologias de software. In: Proceedings of the 20th Brazilian Symposium on Software Engineering, pp. 239-254.

Manikas, K. and Hansen, K. M. (2013). Reviewing the Health of Software Ecosystems - A Conceptual Framework Proposal. In: Proceedings of the 5th International Workshop on Software Ecosystems, pp. 33-44.

Shull, F., Carver, J. and Travassos, G. H. (2001). An Empirical Methodology for Introducing Software Processes. In: Proceedings of European Software Engineering Conference, pp. 288-296.

Villela, K., Travassos, G. H. and Regina, A. (2004). Definição e Construção de Ambientes de Desenvolvimento de Software Orientados a Organização. In: Proceedings of the Brazilian Symposium on Software Quality, pp. 32-46. 